\title{
Diversité Floristique des Ligneux des Systèmes Agroforestiers Cacaoyers du Littoral Cameroun: Cas de l'Arrondissement de Loum
}

\section{Temgoua Lucie Félicité,}

Département de Foresterie, Faculté d'Agronomie et des Sciences Agricoles, Université de Dschang, Dschang Cameroun

\section{Momo Soléfack Marie Caroline,}

Département de Biologie végétale, Faculté de Sciences,

Université de Dschang, Dschang, Cameroun

\section{Boucheké Robert Kévin,}

Département de Foresterie, Faculté d'Agronomie et des Sciences Agricoles,

Université de Dschang, Dschang Cameroun

\section{Doi: 10.19044/esj.2019.v15n9p62ＵRL:http://dx.doi.org/10.19044/esj.2019.v15n9p62}

\section{Résumé}

Les arbres associés dans les systèmes agroforestiers cacaoyers peuvent contribuer à la conservation de la biodiversité. Cependant, ces systèmes sont souvent créés au détriment de la forêt naturelle. Cette étude avait pour but d'évaluer la diversité floristique ligneuse des systèmes agroforestiers cacaoyers dans l'arrondissement de Loum, région du Littoral. Trois zones correspondant à des postes agricoles ont été choisies, il s'agit de Bonkeng, Kwata et Loum Cie. L'inventaire a été réalisé dans 71 parcelles de $2400 \mathrm{~m}^{2}$ et une enquête réalisée auprès des agriculteurs a permis d'identifier l'origine, les différents produits et les usages des arbres associés aux cacaoyers. Un total de 27 espèces appartenant à 23 genres et 18 familles a été inventorié. Les indices de diversité calculés ont comme valeurs moyennes: Shannon $(2,76)$, Pielou $(0,72)$ et Simpson $(0,79)$. La densité des arbres associés aux cacaoyers a été évaluée à 225 tiges/ha avec $89 \%$ d'arbres introduits contre $11 \%$ qui ont été conservés lors de la création de la plantation. Les cinq espèces les plus abondantes ont été Cola acuminata, Elaeis guineensis, Carica papaya, Dacryodes edulis et Persea americana. Les principaux usages de ces arbres sont la production fruitière et l'ombrage fourni aux cacaoyers. Quatre espèces vulnérables suivant les critères de la liste rouge de l'IUCN ont été inventoriées (Entandrophragma cylindricum, Entandrophragma utile, Garcinia kola et Terminalia ivorensis). Ces systèmes agroforestiers cacaoyers ont un bon potentiel de conservation des espèces ligneuses et leur prise en compte dans les mécanismes de paiements 
pour services écosystémiques serait une autre opportunité pour la diversification des revenus des producteurs.

Mots clés: Diversité ligneuse, arbre associé, service écosystémique, cacaoculture, Littoral

\title{
Floristic Diversity of Woody Species in Cocoa Based Agroforestry Systems in Littoral Region of Cameroon: Case of Loum Subdivision
}

\section{Temgoua Lucie Félicité,}

Département de Foresterie, Faculté d'Agronomie et des Sciences Agricoles,

Université de Dschang, Dschang Cameroun

\section{Momo Soléfack Marie Caroline,}

Département de Biologie végétale, Faculté de Sciences,

Université de Dschang, Dschang, Cameroun

\section{Boucheké Robert Kévin,}

Département de Foresterie, Faculté d'Agronomie et des Sciences Agricoles, Université de Dschang, Dschang Cameroun

\begin{abstract}
Associated trees in cocoa agroforestry systems can contribute to biodiversity conservation. However, these systems are often created at the expense of natural forest. This study was carried out with the aim of assessing the woody diversity of cocoa agroforestry systems in Loum Subdivision, Littoral Region. Three sites corresponding to agricultural services were selected: Bonkeng, Kwata and Loum Cie. The inventory was carried out in 71 plots of $2400 \mathrm{~m}^{2}$ and a survey of farmers was done in order to identify the origin, different products and uses of trees associated with cocoa. A total of 27 species belonging to 23 genera and 18 families were inventoried. The biodiversity indices were on average: Shannon (2.76), Pielou (0.72) and Simpson (0.79). The density of associated trees was estimated at $225 \mathrm{stems} / \mathrm{ha}$ with $89 \%$ of introduced trees and $11 \%$, which were preserved during the creation of the plantation. The five most abundant species were Cola acuminata, Elaeis guineensis, Carica papaya, Dacryodes edulis, and Persea americana. The main uses of trees were fruit production and shading provided to cocoa trees. Four vulnerable species following the IUCN red list criteria
\end{abstract}


were inventoried (Entandrophragma cylindricum, Entandrophragma utile, Garcinia kola and Terminalia ivorensis). These cocoa agroforestry systems have good conservation potential of woody species and their taking into account in ecosystem service payments mechanisms would be another opportunity to diversify the farmers'income.

Keywords: Woody diversity, associated trees, ecosystem service, cacaoculture, Littoral

\section{Introduction}

Dans les pays tropicaux, une perte annuelle nette de la superficie forestière entre les années 2000 et 2010 d'environ 7 millions d'hectares et une augmentation des superficies agricoles de 6 millions d'hectare par an a été reportée par FAO (2016). Parmi les facteurs anthropiques entrainant la déforestation dans le bassin du Congo, vient en premier lieu l'agriculture dont l'agriculture itinérante sur brûlis et l'agriculture commerciale incluant la culture du cacao (Sunderlin et al., 2000 ; CARPE, 2005). Celle-ci est suivie par la coupe du bois tant pour les usages domestiques que pour l'approvisionnement du secteur du sciage artisanal et industriel. Les conséquences de la déforestation sont la perte de la biodiversité et la réduction des services écosystémiques comme la séquestration du carbone pour le secteur de l'utilisation des terres et de la foresterie. L'agriculture et le changement d'affectation des terres, représentent respectivement environ 13 et 17 pourcent du total des émissions des gaz à effet de serre résultant de l'activité humaine (FAO, 2008).

L'agroforesterie est un système d'utilisation des terres où des espèces ligneuses pérennes sont délibérément cultivées en association avec des cultures annuelles ou pérennes (Nair, 1993). Elle peut contribuer à résoudre certaines menaces pesant sur l'environnement en particulier dans les pays tropicaux où la destruction des forêts est un enjeu majeur (Torquebiau et al., 2002 ; Woodfine, 2008). Les systèmes agroforestiers jouent un rôle important dans la séquestration du carbone. Ils permettent également grâce à divers produits alimentaires qu'ils fournissent, d'assurer la sécurité alimentaire et d'améliorer les conditions de vie des agriculteurs (Jagoret et al., 2011 ; Nair et Garrity, 2012 ; Atangana et al., 2014).

$\mathrm{Au}$ Cameroun, la cacaoculture pratiquée dans 7 des 10 régions camerounaises est la source principale de revenu pour plus de 400000 familles (Jagoret et Nyasse, 2003). Elle constitue également la principale forme de conversion des écosystèmes naturels (Sonwa et al., 2007 ; Gockowski et Sonwa, 2010). Toutefois, la culture du cacao est faite sous couvert arboré, les agriculteurs conservant ou introduisant dans leurs plantations des espèces ligneuses utiles (Sonwa et al., 2007 ; Gockowski et al., 2010 ; Jagoret, 2011 ; 
Saj et al., 2013 ; Temgoua et al., 2018). Ces systèmes agroforestiers (SAF) cacaoyers ont une structure qui s'apparente à celle des forêts et participent ainsi à la conservation de la diversité ligneuse (Sonwa et al., 2007 ; Saj et al., 2017).

La production cacaoyère de la région du littoral occupe le quatrième rang avec une contribution de 5,87\% à la production nationale. L'essentiel du cacao de la région est fourni par le département du Moungo et plus particulièrement par l'arrondissement de Loum. Le cacao est une culture de rente qui est en expansion dans cet arrondissement et elle se développe essentiellement sous-ombrage. Plusieurs espaces forestiers, classés ou non dans cet arrondissement sont aujourd'hui envahis par les populations à la recherche de terres agricoles. C'est le cas par exemple de la réserve forestière de Loum qui aujourd'hui est complètement envahie par les populations locales qui produisent entre autres du cacao. L'existence de systèmes agroforestiers (SAF) cacaoyers induit le changement des modes d'occupation des sols, mais aussi des services écosystémiques et socio- économiques rendus par les écosystèmes naturels qui les précédaient.

Le potentiel de conservation de la biodiversité de ces systèmes agroforestiers offre un avantage certain dans leur prise en compte dans le paiement pour services écosystémiques dans le cadre de la Convention Cadre des Nations Unies contre le Changement Climatique. Il serait donc important d'avoir des informations suffisamment précises sur la diversité des ligneux et les stocks de carbone de ces SAF. Si dans d'autres bassins de production du Cameroun, plusieurs études ont déjà été menées sur les potentialités de conservation de la biodiversité des systèmes agroforestiers cacaoyers (Jagoret, 2011 ; Mbolo et al., 2016 ; Zapfack et al., 2016 ; Madountsap et al., 2017; Saj et al., 2017), ce n'est pas encore le cas pour le bassin du Littoral. Cette étude a donc comme objectif global d'évaluer la contribution des systèmes agroforestiers cacaoyer à la conservation de la biodiversité dans le bassin de production du Littoral avec un focus sur l'Arrondissement de Loum. Les objectifs spécifiques de cette étude sont : (1) analyser la diversité et la structure de la flore ligneuse dans ces systèmes agroforestiers cacaoyers et (2) Identifier les usages des arbres associés.

\section{Matériel et Méthodes Site de l'étude}

L'étude a été menée dans l'Arrondissement de Loum, Département du Moungo (figure 1). Ce département est situé dans la zone agro-écologique de forêt dense humide à pluviométrie monomodale. Le département est caractérisé par un climat tropical humide: une saison des pluies allant de mars à novembre et une saison sèche allant de décembre à février. La pluviométrie moyenne est de $2700 \mathrm{~mm}$ et les températures varient de $22,4{ }^{\circ} \mathrm{C}$ à $27,5^{\circ} \mathrm{C}$ en 
fonction de l'altitude (Martin et Sieffermann, 1966). Les sols sont d'origine volcanique, de texture noire constitué par un mélange de quartz et d'argile très meuble, propice à l'agriculture dont est essentiellement basée l'économie du département. Le Moungo est utilisé pour la culture intensive de la banane depuis les années 1950 (Delvaux, 1988). La végétation est dominée par la forêt humide, les plantations de cacaoyers et de caféiers, le palmier à huile, les arbres fruitiers et les plantations industrielles de banane. L'arrondissement de Loum abrite la réserve forestière de Loum. Cette réserve d'une superficie de 1100 ha créée en 1932 est aujourd'hui envahie par les populations locales qui y pratiquent de l'agriculture.

\section{Méthodes de collecte et d'analyse des données}

L'étude a été menée dans trois localités, Kwata, Bonkeng et Loum compagnie (Loum Cie). La localité de Kwata inclut la réserve forestière de Loum. Les critères de sélection des SAF cacaoyers ont été la superficie $(\geq 0,5$ ha) et l'exploitation en phase de production.

La mise en place des placettes de comptage a été faite en adaptant la méthode décrite par Hairiah et al., 2011) avec l'installation des placettes principales de forme rectangulaire de $2400 \mathrm{~m}^{2}(40 \mathrm{~m} \times 60 \mathrm{~m})$ pour le comptage des ligneux ayant un diamètre à hauteur de poitrine supérieur ou égal à $30 \mathrm{~cm}$ et des placettes secondaires de $800 \mathrm{~m}^{2}(20 \mathrm{~m} \times 40 \mathrm{~m})$ pour les ligneux ayant un diamètre compris entre 5 et $30 \mathrm{~cm}$. Au total, 71 placettes ont été installées. L'inventaire a pris en compte la composition floristique (différentes espèces présentes dans les parcelles) et le diamètre à hauteur de poitrine. La nomenclature botanique utilisée est celle adoptée par Lebrun et Stork (1991, 1992, 1995, 1997). Les pieds de cacaoyers ont été également comptés et les diamètres mesurés. Des entretiens semi-structurés avec 25 agriculteurs (propriétaires et/ou gestionnaires) des champs ont permis d'obtenir des informations sur le mode de gestion des cacaoyères, l'origine (conservé ou introduit) et les usages des arbres associés. 


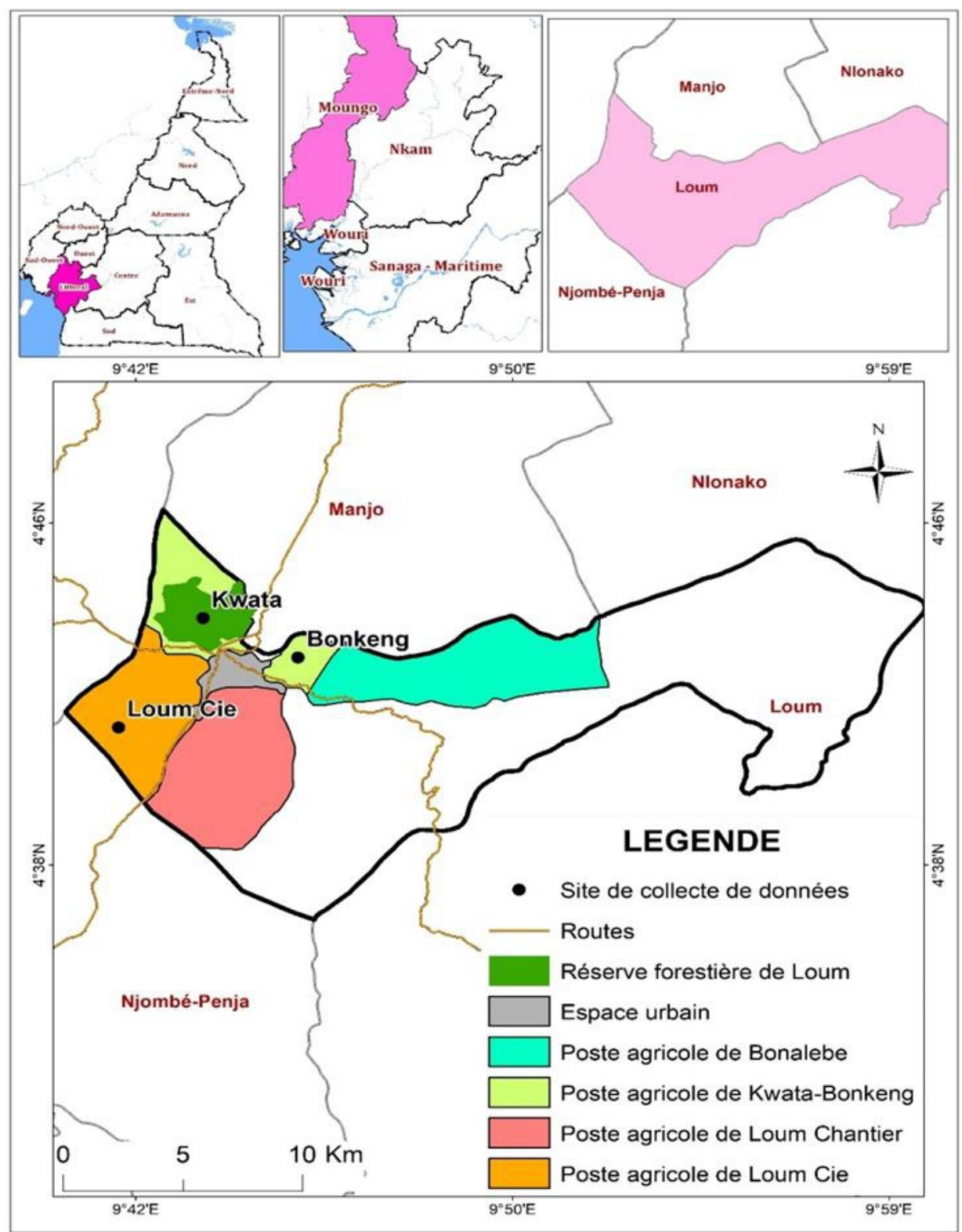

Figure 1 : Localisation de l'arrondissement de Loum dans la région du Littoral

La caractérisation floristique des systèmes agroforestiers s'est faite par la détermination des paramètres tels que : la richesse spécifique, l'abondance des espèces et des familles, la densité du peuplement d'arbres et sa structure diamétrique.

La richesse spécifique correspond au nombre d'espèces que compte une communauté ou un peuplement (Ramade, 1994). Le nombre de familles 
et le nombre d'espèces présentes dans chaque parcelle a été déterminé ainsi que le nombre d'individus de chaque espèce.

L'abondance d'une espèce ou d'une famille dans une communauté végétale donnée est l'importance numérique des individus de cette espèce ou famille dans la communauté par rapport au nombre total d'individus. L'abondance relative s'exprime en pourcentage :

$$
\mathbf{A r}=\frac{\mathbf{n i}}{\mathbf{N}} \times 100
$$

Où : Ar est l'abondance relative (en \%), ni est le nombre d'individus d'une espèce $\mathrm{i} ; \mathrm{N}$ le nombre total d'individus de toutes les espèces.

L'indice de diversité de Shannon-Weaver $\left(\mathrm{H}^{\prime}\right)$ permet de mesurer les possibilités d'interaction entre les espèces qui composent une communauté. Cet indice tient compte du nombre d'espèces présentes et de la répartition des individus au sein de ces espèces.

$$
\mathbf{H}^{\prime}=-\sum(\mathbf{n i} / \mathbf{N}) \log _{2}(\mathbf{n i} / \mathbf{N})
$$

L'équitabilité de Pielou $(\mathrm{E})$ exprime la régularité, la répartition équitable des espèces au sein d'une communauté. Cet indice qui varie de 0 à 1 est maximal quand les espèces ont des abondances identiques dans le peuplement et il est minimal quand une seule espèce domine tout le peuplement.

$$
\mathbf{E}=\mathbf{H}^{\prime} / \log _{2} \mathbf{S}
$$

$\mathrm{S}$ est le nombre total d'espèces relevées et $\mathrm{H}^{\prime}$ l'indice de Shannon.

L'indice de diversité de Simpson (D') : c'est la probabilité pour que deux individus tirés au hasard soient d'espèces différentes. Il est représenté par la réciproque de l'indice de Simpson (D). Le maximum de diversité est représenté par la valeur 1 et le minimum de diversité par la valeur 0 .

$$
D^{\prime}=1-\sum(\mathbf{n i} / \mathbf{N})^{2}
$$

L'Indice de Jaccard (J) : Il traduit la similarité qui existe entre les groupements végétaux et a permis de faire une comparaison de similarité entre les systèmes agroforestiers cacaoyers dans différents sites. Cet indice se calcule selon la formule qui suit:

$$
\mathbf{J}=\frac{\mathbf{C}}{\mathbf{A}+\mathbf{B}-\mathbf{C}}
$$

Où $\mathrm{A}$ : désigne l'ensemble des espèces du site $\mathrm{A}$; $\mathrm{B}$ désigne l'ensemble des espèces du site $\mathrm{B}$ et $\mathrm{C}$ désigne les espèces communes aux deux sites en comparaison.

La densité des espèces a été estimée par la formule de Kent et Coker (1992). La densité d'une espèce (D) est le nombre d'individus de cette espèce à l'hectare. Elle est évaluée par la formule :

$$
\mathbf{D}=\mathbf{n} / \mathbf{S}
$$


Avec : D la densité (en tiges/ha), n le nombre de tiges présentes sur la surface considérée et $\mathrm{S}$ la surface considérée (ha).

La structure horizontale ou diamétrique du peuplement ligneux : les ligneux ont été répartis dans les différentes classes de diamètre d'amplitude $10 \mathrm{~cm}$, suivant ainsi les recommandations de Farichon et al. (1998) qui préconisent de choisir pour des raisons pratiques, des classes de tailles constantes de largeur égale à 5 ou $10 \mathrm{~cm}$. Douze classes de diamètres ont été définies. Les limites de ces classes ont été les suivantes : ]5-15 cm], ]15-25 $\mathrm{cm}]$, ]25-35 cm], ]35-45 cm], ]45-55 cm], ]55-65 cm], ]65-75 cm], ]75-85 cm] , ]85-95 cm], ]95-105], ]105-115 cm] et $>115 \mathrm{~cm}$.

La distribution par classe d'âge : trois classes d'âges ont été définies pour les plantations cacaoyères d'après la catégorisation de Saj et al. (2017): la classe «Immature », pour les plantations de moins de 11 ans; la classe « Jeune » pour les plantations d'âges compris entre 11 et 20 ans et la classe « Mature », comprenant les plantations d'âges compris entre 21 et 40 ans.

\section{Analyse statistique}

Le logiciel Microsoft Excel 2016 a servi au classement des données numériques et à l'élaboration des divers graphiques. Le logiciel XLSTAT 2018.1. a été utilisé pour le test d'analyse de la variance (ANOVA) afin de déterminer si le site à un effet sur les paramètres de richesse spécifique et de densité des arbres. Lorsque les différences étaient significatives, les moyennes étaient séparées par le test de Duncan au seuil de significativité de 5\%.

\section{Résultats}

\section{Caractéristiques floristiques et richesse spécifique}

Dans l'ensemble des 71 parcelles agroforestières étudiées, le cortège floristique fait état de 27 espèces appartenant à 23 genres et 18 familles. En ce qui concerne les abondances relatives, la famille des Sterculiaceae (figure 2) est la plus représentée en nombre d'individus (19,37\% des individus), suivie des Arecaceae (12,87\%) et des Caricaceae (8,57\%). Onze familles ont chacune une abondance relative inférieure à $5 \%$. En terme du nombre d'espèces deux familles occupent la première place avec trois espèces chacune (Fabaceae et Rutaceae); quatre familles ont deux espèces chacune (Sterculiaceae Combretaceae, Meliaceae et Arecaceae) et les 12 autres n'en possèdent chacune qu'une seule. 


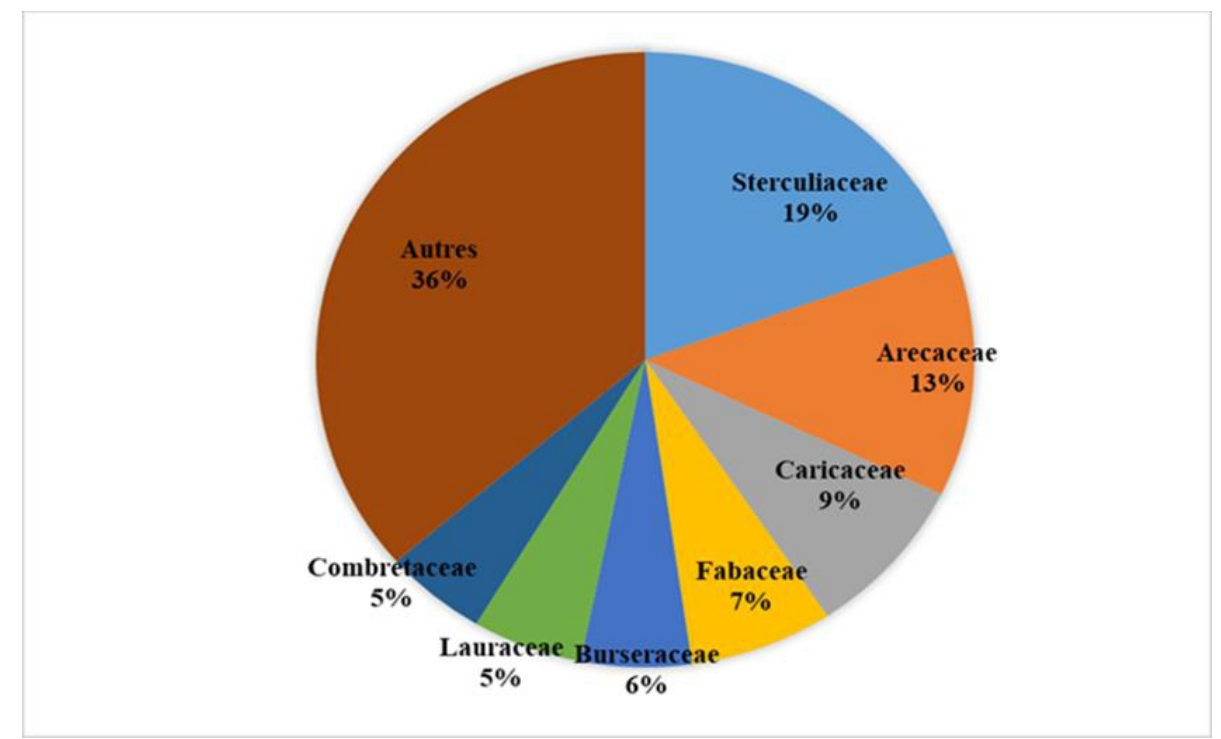

Figure 2: Abondance relative des familles des ligneux associés

L'espèce ligneuse la plus abondante est Cola acuminata (Kolatier) avec 21,78\% du total des individus, suivie de Elaeis guineensis (Palmier à huile), Carica papaya (Papayer) et Dacryodes edulis (Safoutier) (figure 3). Douze espèces ont une abondance relative inférieure à $1 \%$. Les espèces les moins abondantes sont Annona muricata (Corossolier) avec 0,11\% des individus, Picralima nitida (Ebam) avec $0,11 \%$ et Citrus maxima (Pamplemoussier) avec 0,06\%.

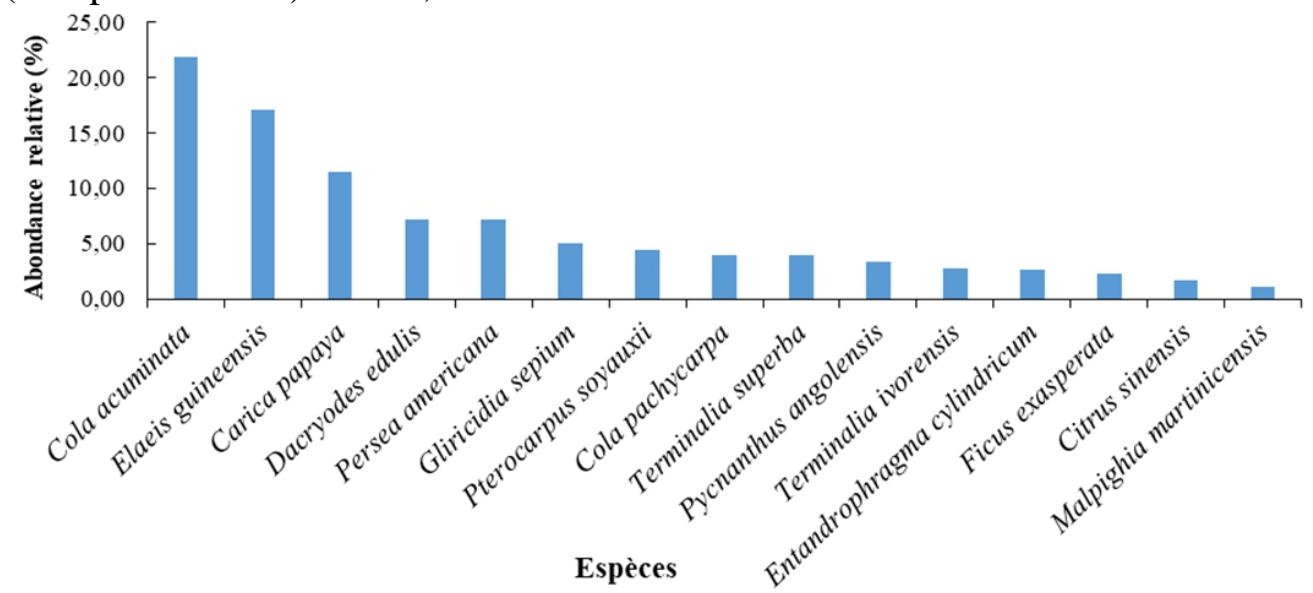

Figure 3 : Abondance relative des espèces ligneuses ayant plus de $1 \%$ des individus

Concernant le statut de conservation suivant les critères de la liste rouge des espèces menacées de l'UICN (2018), 4 espèces inventoriées sont vulnérables. Il s'agit de: Entandrophragma cylindricum (Sapelli), Entandrophragma utile (Sipo), Garcinia kola (Bitacola) et Terminalia 
ivorensis (Framiré). Ces espèces sont faiblement représentées dans les cacaoyères avec des abondances relatives de $2,66 \%, 0,27 \%, 0,17 \%$ et $0,17 \%$ respectivement. Le tableau 1 présente la richesse spécifique et les indices de diversité des SAF par site.

Tableau 1 : Richesse spécifiques et indices de diversité des SAF cacaoyers

\begin{tabular}{lrrrrrr}
\hline Sites & $\begin{array}{r}\text { Nombre } \\
\text { placettes }\end{array}$ & $\begin{array}{r}\text { Nombre } \\
\text { espèces }\end{array}$ & $\begin{array}{r}\text { Nombre } \\
\text { familles }\end{array}$ & $\begin{array}{r}\text { Indice de } \\
\text { Shannon }\end{array}$ & $\begin{array}{r}\text { Indice de } \\
\text { Simpson }\end{array}$ & $\begin{array}{r}\text { Equitabilité } \\
\text { de Pielou }\end{array}$ \\
\hline Bonkeng & 27 & 11 & 9 & 2,48 & 0,77 & 0,72 \\
Kwata & 21 & 17 & 13 & 3,55 & 0,90 & 0,87 \\
Loum Cie & 23 & 14 & 12 & 2,27 & 0,71 & 0,59 \\
Arrondisse & 71 & 27 & 18 & 2,76 & 0,79 & 0,72 \\
ment Loum & & & & & & \\
\hline
\end{tabular}

Le tableau 1 montre que le nombre d'espèces ligneuses associées dans les cacaoyères varie de 11 à 17 . La plus grande richesse spécifique a été observée dans les cacaoyères de Kwata et la plus faible à Bonkeng. L'indice de Shannon qui prend en compte les proportions représentées par chaque espèce, avec des valeurs comprises entre 2,27 et 3,55 indique une diversité ligneuse moyenne, avec néanmoins une meilleure diversité dans la localité de Kwata. L'indice de Simpson dans cette localité est également le plus proche de un $(0,90)$, traduisant une plus grande diversité par rapport aux autres zones de l'étude. L'équirépartition des espèces est plus faible dans la zone de Loum Cie $(0,59)$, ce qui traduit un déséquilibre de ces systèmes et une mauvaise répartition des espèces, avec des espèces dominantes. En effet, dans cette zone, Cola acuminata et Carica papaya représentent à elles seules $74,7 \%$ des individus recensés (figure 4).

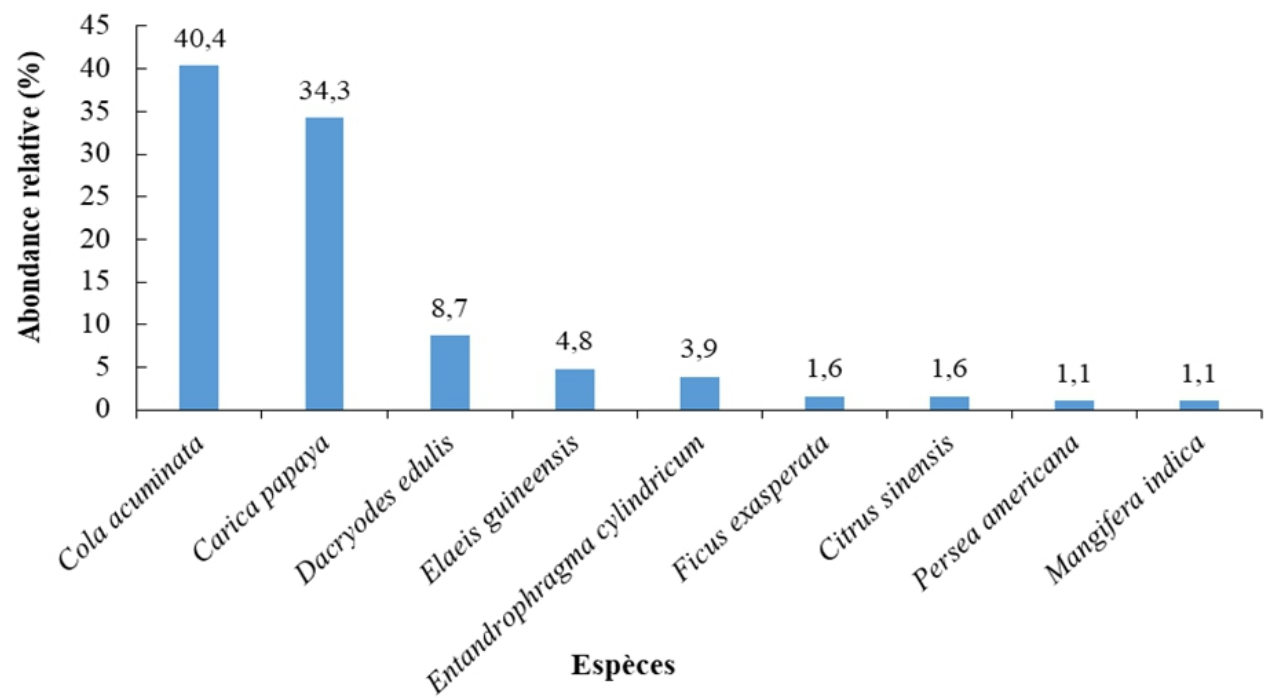

Figure 4 : Abondance relative des espèces ayant plus de 1\% des individus à Loum Cie 
À Bonkeng (figure 5), les espèces les plus abondantes sont Elaeis guineensis (35,5\% des individus recensés dans la zone), Cola acuminata $(23,6 \%)$ et Gliricidia sepium (14,9\%).

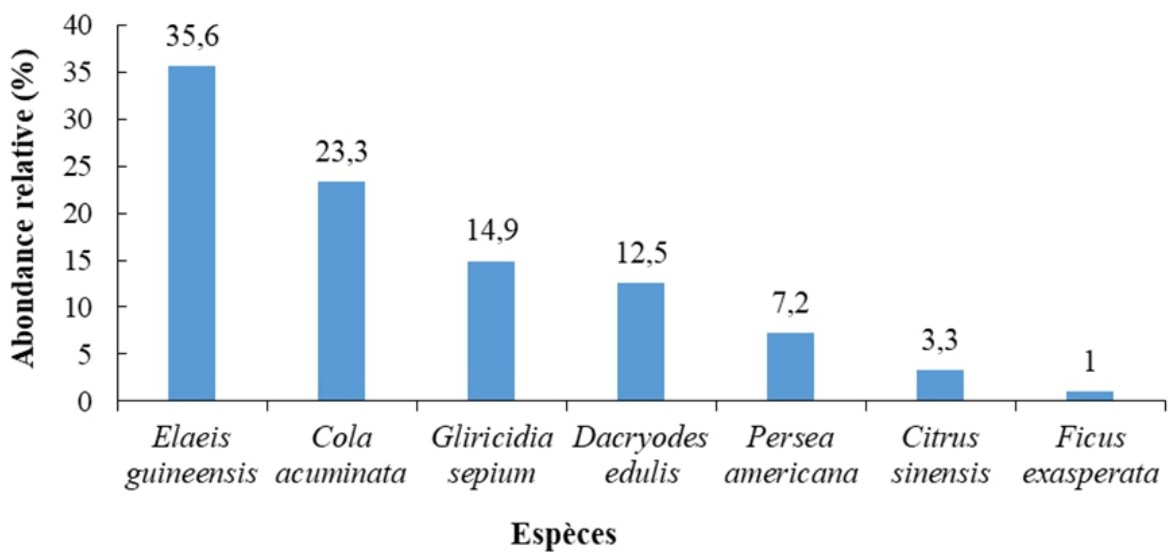

Figure 5 : Abondance relative des espèces ayant plus de $1 \%$ des individus à Bonkeng

Dans la zone de Kwata où la répartition des individus entre les espèces est la plus élevée, ce sont Persea americana (Avocatier), Pterocarpus soyauxii (Padouk rouge) et Cola pachycarpa (Cola du Singe) qui sont les espèces les plus abondantes (figure 6).

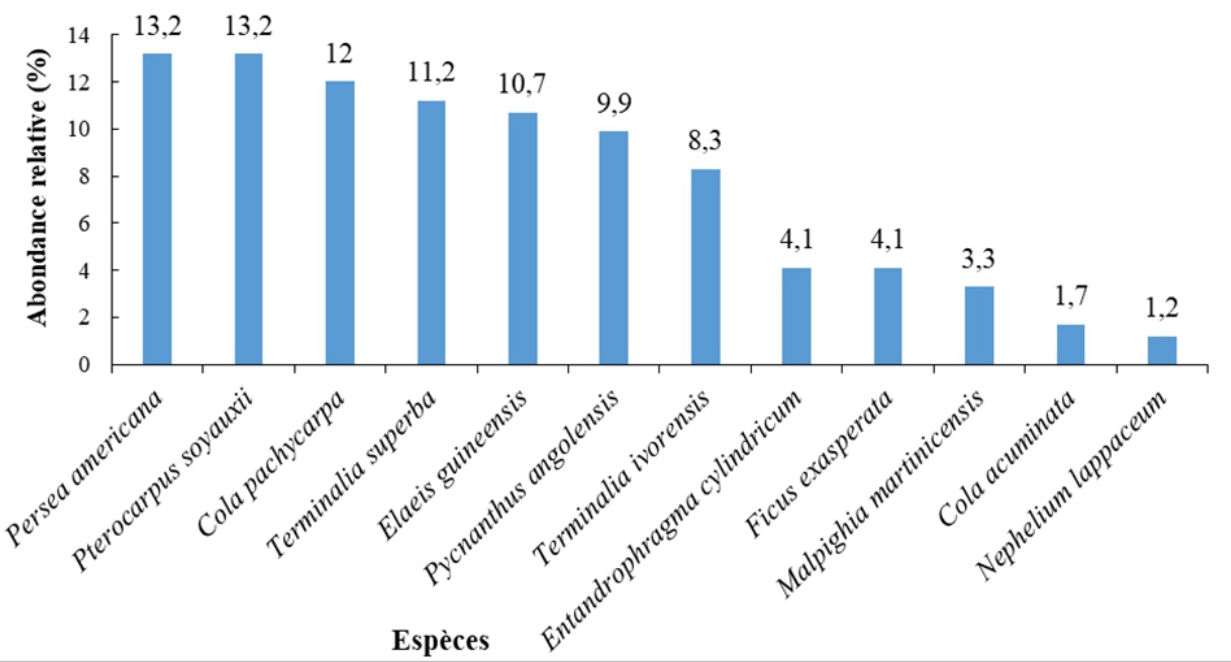

Figure 6: Abondance relative des espèces ayant plus de $1 \%$ des individus à Kwata

\section{Similitude entre les systèmes agroforestiers}

Les cortèges floristiques des cacaoyères des trois localités présentent peu de similitude (tableau 2). 
Tableau 2 : Indices de similitude de Jaccard entre les SAF des différents sites

\begin{tabular}{lccc}
\hline \multicolumn{1}{c}{ Sites } & \multicolumn{3}{c}{ Indices de Jaccard (J) } \\
\cline { 2 - 4 } & Bonkeng & Kwata & Loum Cie \\
\hline Bonkeng & 1 & 1 & \\
Kwata & 0,27 & 0,24 & 1 \\
Loum Cie & 0,39 & & \\
\hline
\end{tabular}

Les indices de similitude de Jaccard entre les localités varient de 0,24 à 0,39 . Ces valeurs inférieures à 0,5 signifient que les fonds floristiques des systèmes agroforestiers cacaoyers de ces trois localités ne sont pas proches. Les SAF de Kwata présentent le moins d'espèces communes avec ceux des autres localités. Par contre ceux des localités de Bonkeng et Loum Cie ont plus d'espèces communes entre eux $(\mathrm{J}=0,39)$. Seulement 5 espèces se sont retrouvées dans toutes les trois localités. Il s'agit de Cola acuminata, Elaeis guineensis, Dacryodes edulis, Persea americana et Ficus exasperata. Neuf espèces ont été identifiées exclusivement dans les cacaoyères de la zone de Kwata. Il s'agit pour la plupart des espèces forestières qui ont été utilisées par les services forestiers pour le reboisement de la réserve forestière de Loum qui se trouve dans ce site. Quelques-unes de ces espèces sont Pterocarpus soyauxii, Pycnanthus angolensis (Ilomba), Terminalia ivorensis et Entandrophragma utile.

\section{Densité des arbres et des cacaoyers}

Les SAF cacaoyers étudiés ont une densité moyenne de 225 arbres associés par hectare, et une densité de cacaoyers évaluée à 1080 individus à l'hectare. Le tableau 3 montre que la densité de cacaoyers varie de 800 à 1426 tiges/ha. Les différences de densité cacaoyère sont significatives entre toutes les zones $(\mathrm{p}<0,030)$.

Tableau 3: Densité des arbres et des cacaoyers dans les systèmes agroforestiers

\begin{tabular}{ccc}
\hline Sites & \multicolumn{2}{c}{ Densité (tige/ha) } \\
\cline { 2 - 3 } & Cacaoyers & Ligneux associés \\
\hline Bonkeng & $800 \mathrm{a}$ & $167 \mathrm{a}$ \\
Kwata & $1014 \mathrm{~b}$ & $183 \mathrm{a}$ \\
Loum Cie & $1426 \mathrm{c}$ & $325 \mathrm{~b}$ \\
Moyenne & 1080 & 225 \\
arrondissement & &
\end{tabular}

Les valeurs suivies par une même lettre dans une colonne ne sont pas significativement différentes au seuil de $5 \%$

\section{Structure diamétrique des arbres associés}

Les peuplements d'arbres associés des cacaoyères étudiées présentent une structure diamétrique exponentielle décroissante sous forme de $\mathrm{J}$ inversé à Bonkeng, tandis qu'à Kwata et Loum Cie la courbe a une forme en cloche (figure 7). 


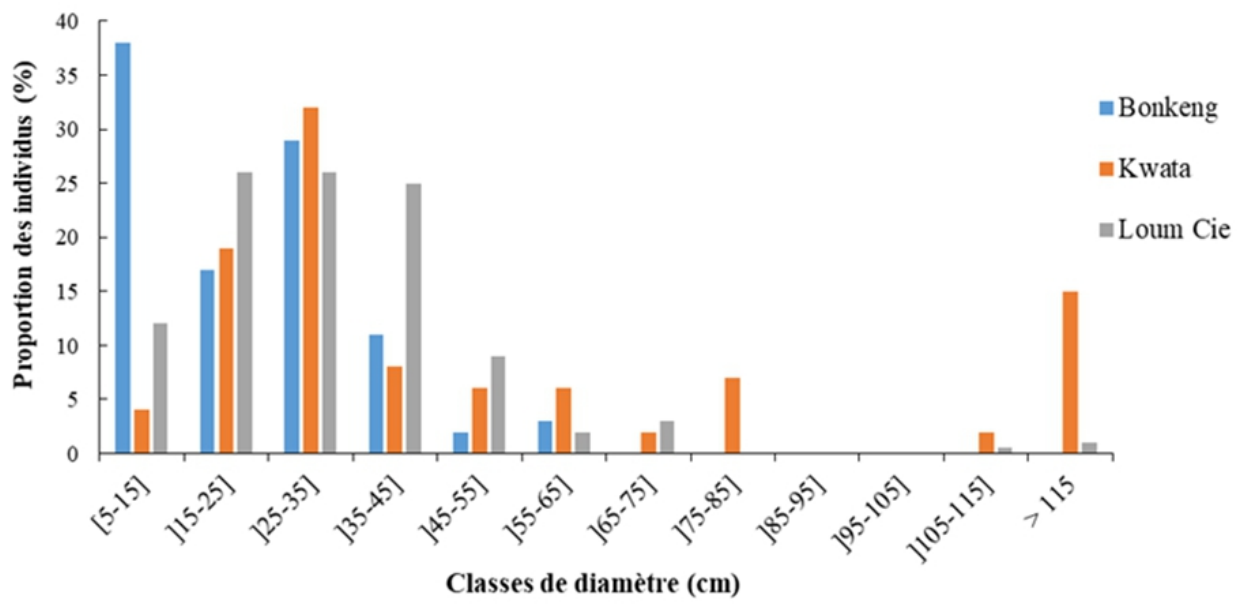

Figure 7 : Structure diamétrique des arbres associés dans les systèmes agroforestiers

À Bonkeng on note une forte proportion des arbres ayant un diamètre compris entre 5 et $15 \mathrm{~cm}(38 \%)$, les plus faibles valeurs dans cette classe de diamètre étant rencontrées dans la localité de Kwata (4\%). A Bonkeng tous les arbres ont un diamètre inférieur à $65 \mathrm{~cm}$. A Loum Cie on note une très faible proportion d'arbres de diamètre supérieur à $65 \mathrm{~cm} \mathrm{(4,5 \% ),} \mathrm{tandis} \mathrm{que} \mathrm{cette}$ proportion est de $24 \%$ dans la zone de Kwata. Les arbres de faible diamètre représentent la jeune régénération, généralement plantés par les agriculteurs. Ceux de plus grands diamètres ont quant à eux été conservés au moment de la création de la plantation cacaoyère.

\section{Age des cacaoyères}

Les enquêtes menées auprès des propriétaires des SAF ont permis d'établir une classification de ces derniers par classes d'âge. La majorité des SAF sont jeunes, avec un âge compris entre 11 et 20 ans. Les plantations d'âge compris entre 20 et 40 ans, dites Matures, sont moins nombreuses, tandis qu'une faible proportion est constituée des plantations d'âges inférieurs à 11 ans, dites immatures (figure 8). 


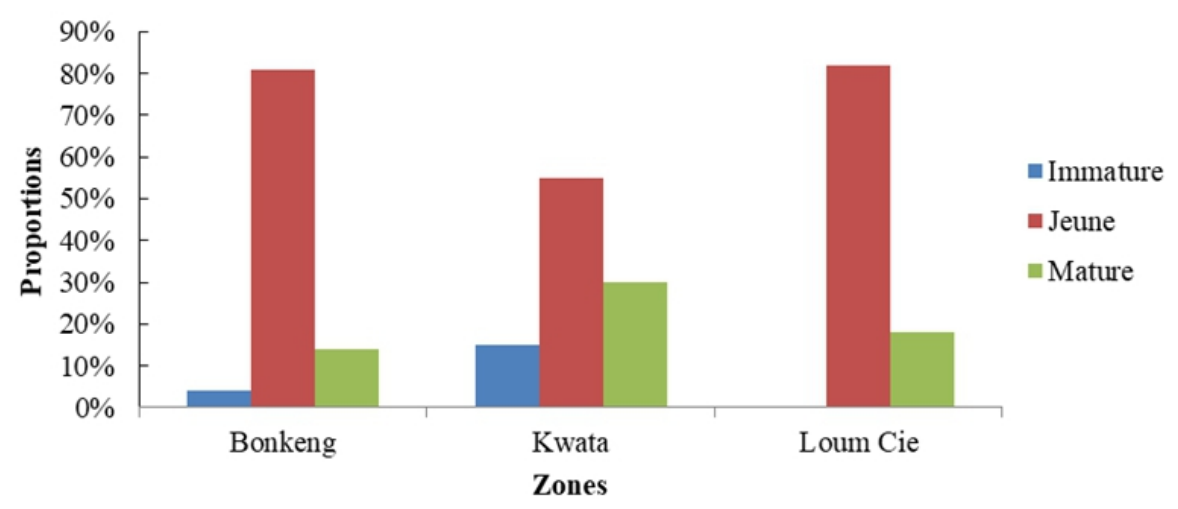

Figure 8 : Répartition des systèmes agroforestiers cacaoyers par catégories d'âge

\section{Statut et usages des ligneux associés}

La figure 9 présente les proportions d'arbres associés préservés et introduits dans les cacaoyères dans différentes localités.

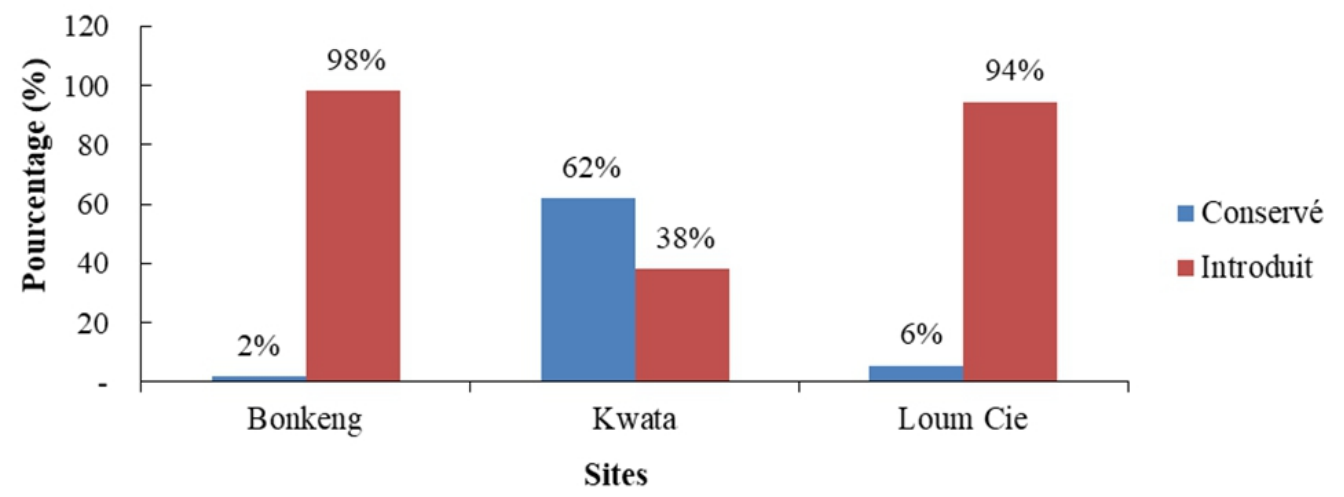

Figure 9 : Proportions des espèces ligneuses introduites et conservées dans les systèmes agroforestiers

Dans l'ensemble de la zone d'étude, les arbres associés aux SAF cacaoyers sont à $89 \%$ des arbres introduits contre seulement $11 \%$ d'arbres conservés. Dans la zone de Bonkeng et de Loum Cie, les proportions d'arbres conservés (2\% et $6 \%$ respectivement) sont très inférieures aux proportions d'arbres introduits (plus de 94\%). Ces proportions diffèrent significativement de celles de la zone de Kwata, où les proportions d'arbres introduits (62\%) sont en deçà des proportions d'arbres conservés (38\%).

Trois grands groupes d'usages des arbres associés ont été répertoriés : l'ombrage, la production fruitière et l'utilisation des arbres comme tuteurs pour la culture du poivre. La figure 10 présente les proportions d'arbres associés aux cacaoyers dans les SAF en fonction des usages prioritaires qui en sont faits. 


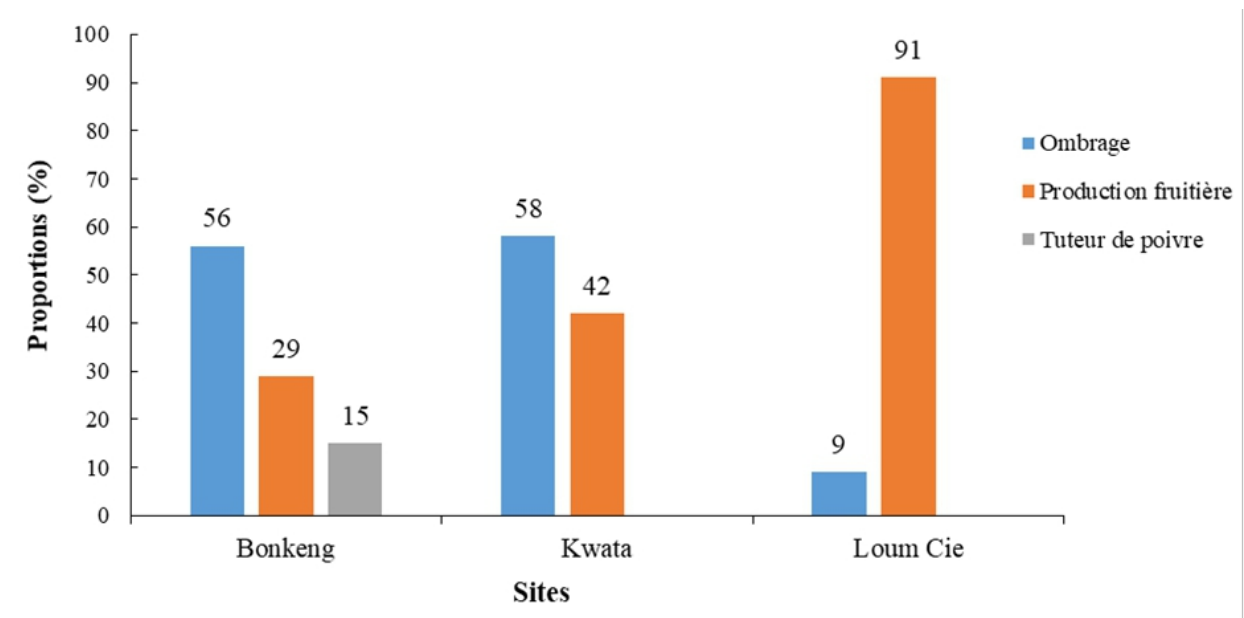

Figure 10: Proportion des espèces associées en fonction des usages par site

Il ressort de la figure 10 que dans les zones de Bonkeng et de Kwata, plus de la moitié des agriculteurs associent les arbres à la cacaoculture principalement pour l'ombrage, ce qui n'est pas le cas dans la zone de Loum Cie, où les arbres associés servent majoritairement à la production fruitière pour $91 \%$ des enquêtés. Bonkeng est la seule localité où les arbres associés sont utilisés prioritairement comme tuteurs par $15 \%$ des agriculteurs. Il s'agit en effet de Gliricidia sepium utilisé comme tuteur pour la culture du poivre (Piper nigrum). Les ligneux les plus utilisés pour fournir de l'ombre aux cacaoyers sont Entandrophragma cylindricum, Ficus exasperata, Cola acuminata, Pterocarpus soyauxii et Terminalia superba (Fraké). Quant à la production fruitière elle est essentiellement assurée par Cola acuminata, Carica papaya, Persea americana, Elaeis guineensis et Dacryodes edulis.

\section{Discussion}

\section{Structure et composition floristique des SAF}

Un total de 27 espèces ligneuses appartenant à 18 familles a été répertorié dans les systèmes agroforestiers cacaoyers de l'arrondissement. Cette richesse spécifique est proche de celles de 26 et 21 espèces trouvées respectivement par Manfo et al. (2015) et Madountsap et al. (2017) dans les cacaoyères de la zone de transition forêt savane de la région du Centre Cameroun. Cette similitude peut s'expliquer par les objectifs des agriculteurs. Dans notre zone d'étude tout comme dans ces zones de transition forêt savane du Centre, les agriculteurs ne conservent ou alors n'introduisent dans leurs cacaoyères que des espèces qui leur sont vraiment utiles, ce choix étant guidé par les opportunités commerciales des espèces.

La richesse spécifique trouvée dans notre étude est inférieure à celle trouvée par Jagoret et al. (2014) et Temgoua et al. (2018) qui était de 64 et 72 
espèces respectivement à Zima dans le Centre et à Belabo à l'Est Cameroun. Cette différence pourrait s'expliquer par le type de végétation dans ces zones écologiquement différentes (zone forestière monomodale et zone forestière bimodale) qui aurait une influence sur la diversité floristique, mais aussi par les objectifs des agriculteurs de la zone forestière bimodale qui créent des agroforêts cacaoyères complexes dans lesquelles ils conservent plusieurs espèces d'arbres.

Les indices de diversité de Shannon de 2,48 et 2,27 trouvés dans les sites de Bonkeng et Loum Cie sont similaires à ceux de 2,42 et 2,68 trouvés par Jagoret et al. (2014) et Madountsap et al. (2017) respectivement. Ces indices sont supérieurs à ceux de 1,55 trouvés par Kpangui et al. (2015) au Centre de la Côte d'Ivoire. Kwata a été la zone ayant la plus grande diversité et son indice de Shannon de 3,55 est similaire à celui de Sonwa et al. (2007) et Zapfack et al. (2016) dans les cacaoyères du centre et du Sud Cameroun. Les indices de Simpson $(0,79)$ et de Pielou $(0,72)$ obtenus montrent une bonne répartition des individus au sein des espèces avec néanmoins une plus grande abondance pour Cola acuminata, Elaeis guineensis, Carica papaya, Dacryodes edulis et Persea americana.

Le coefficient de similitude de Jaccard des différentes zones de l'étude est inférieur à 0,4 . Cette faible similitude peut s'expliquer par des préférences différentes des agriculteurs pour les espèces dans ces différentes zones. En effet, après la production du cacao, les agriculteurs donne la priorité à un faible nombre d'espèces d'arbres qu'ils introduisent/ conservent dans leurs cacaoyères. Dans la zone de Loum Cie après le cacao, la priorité est donnée à la noix de Cola (Cola acuminata), à la papaye (Carica papaya) et au Safou (Dacryodes edulis). Dans la zone de Bonkeng la priorité est donnée au palmier à huile (Elaeis guineensis), à la noix de cola (Cola acuminata) et à Gliricidia sepium utilisé comme tuteur pour la culture du poivre. Dans la zone de Kwata la priorité est donnée à la production de l'avocat (Persea americana). Kwata a la plus faible similitude avec les deux autres zones. En effet, dans cette zone qui abrite la réserve forestière de Loum, les agriculteurs n'ont pas vraiment la liberté d'abattre les espèces forestières qui s'y trouvent. Cette contrainte empêche donc véritablement les agriculteurs d'introduire beaucoup d'autres nouvelles espèces, sinon l'ombrage dans les cacaoyères serait excessif. Ainsi, les arbres sont le plus souvent ceux qui ont été conservés (62\%) lors de la création de la cacaoyère contrairement aux zones de Bonkeng et de Loum Cie où plus de $90 \%$ des arbres ont été introduits.

La densité moyenne des arbres associés (225 arbres/ha) est similaire à celles de 233 et 244 arbres/ha obtenues par Saj et al. (2013) à Ngomedzap et Bokito au centre Cameroun. Elle est cependant supérieure aux valeurs obtenues par Norgrove et Hauser (2013) à Zoatoupsie au Sud Cameroun (115 arbres/ha) et celle obtenue par Madountsap et al. (2017) à Talba au Centre 
Cameroun (77 arbres /ha). En considérant séparément les trois zones de notre étude, on observe une différence significative entre la densité des arbres associés de la zone de Loum Cie (325 arbres/ha) avec celle de Bonkeng (167) et Kwata (183). Cette différence traduit une certaine différence dans les techniques culturales des paysans dans ces localités. Dans la zone de Loum $\mathrm{Cie}$, les agriculteurs introduisent une forte proportion de Carica papaya dans les cacaoyères. Le papayer ne produisant pas beaucoup d'ombrage, les agriculteurs peuvent donc introduire un plus grand nombre de tiges à l'hectare. Dans la zone de Kwata, dans la réserve de Loum, la culture du cacao est faite sous le couvert des arbres forestiers. Ici en raison des contrôles des services forestiers, les agriculteurs n'ont pas beaucoup de possibilités de couper ou d'introduire de nouveaux arbres.

En ce qui concerne la distribution des arbres par classes de diamètre, les proportions d'individus diminuent avec l'accroissement du diamètre. Les classes de diamètre ayant les plus grands effectifs vont de 5 à $35 \mathrm{~cm}$. Cette analyse permet de rendre compte du pouvoir de régénération assez important des ligneux dans les SAF. Dans d'autres études similaires (Noiha et al., 2015 ; Zapfack et al., 2016 ; Madountsap et al., 2017), la structure diamétrique des arbres associés était également exponentielle décroissante avec une prédominance des arbres de petits diamètres $(\leq 40 \mathrm{~cm})$ tout comme ce que nous avons trouvé dans la zone de Bonkeng. La zone de Kwata incluse dans la réserve forestière de Loum présente cependant une courbe de distribution en cloche, la plus faible proportion d'arbres de diamètre inférieur à $15 \mathrm{~cm}(4 \%)$, dénotant une régénération plutôt faible. Elle présente également la plus grande proportion d'arbres de plus de $65 \mathrm{~cm}$ de diamètre (24\%). En effet, le mode de tenure des terres au sein de la réserve n'encourage pas les agriculteurs d'y planter des arbres. De même, les patrouilles du service forestier, sans véritablement supprimer l'abattage des essences forestières, ralentissent néanmoins considérablement leur exploitation.

\section{Usages des arbres associés}

Dans les cacaoyères, les agriculteurs conservent et introduisent des arbres qui leur sont utiles. Ce qui corroborent les observations de plusieurs auteurs au Cameroun (Sonwa et al., 2007 ; Gockowski et al., 2010 ; Saj et al., 2017 ; Temgoua et al., 2018) et en Côte d'Ivoire (Kpangui et al., 2015 ; Cissé et al., 2016). Trois usages prioritaires des arbres ont été évoqués par les agriculteurs interviewés dans l'Arrondissement de Loum : Usage à des fins de production fruitière, usage pour fourniture d'ombrage, et un dernier usage comme tuteur de poivre. Ce dernier usage est propre à la zone de Bonkeng et l'espèce utilisée est Gliricidia sepium. En effet l'arrondissement de Loum est inclus dans la zone géographique de la culture du poivre de Penja (première Indication Géographique Protégée en Afrique sub-saharienne), qui est en 
pleine expansion dans le département du Moungo. Compte tenu de la rareté des terres arables, on note la conversion progressive d'une petite proportion de SAF cacaoyers de la zone en plantations de poivrier. Parmi les principales espèces introduites dans les cacaoyères pour la production fruitière figure Carica papaya. Des études similaires dans d'autres régions du Cameroun, n'ont pas mentionné une abondance de cette espèce dans les SAF cacaoyers. Cette abondance dans notre zone d'étude peut s'expliquer par le fait que le département du Moungo est le principal bassin de production de la papaye solo au Cameroun.

Dans des études similaires (Saj et al., 2017 ; Temgoua et al., 2018), les arbres associés fournissent plus de trois usages : production alimentaire, usages médicinaux, fourniture du bois, amélioration de la fertilité du sol et fourniture d'ombrage aux cacaoyers. Cette différence s'expliquerait par le refus des agriculteurs (69\%) de notre zone d'étude d'introduire des arbres autres que fruitiers dans les SAF cacaoyers ; couplé au mode de gestion de l'ombrage tourné vers la suppression des arbres associés. Selon les agriculteurs, les contraintes liées à l'introduction/conservation d'arbres associés dans les SAF cacaoyers sont : la tenure foncière (nombreux sont ceux qui ne sont que locataires des champs) et le manque de plants. Le manque de plants est aussi l'une des contraintes à la plantation des arbres soulevées par les agriculteurs à l'Est Cameroun (Temgoua et al., 2018).

Il est à noter que le choix de conserver ou d'introduire des arbres dans les SAF cacaoyers, est aussi guidé par les opportunités de marché qui s'offrent aux agriculteurs de l'Arrondissement de Loum, notamment par la présence du principal axe économique du pays (Bafoussam-Douala). Ainsi, pour combler les déficits de production cacaoyère ou pour rendre l'afflux des revenus d'origine agricole constant, ces agriculteurs façonnent leurs SAF en donnant la priorité à la conservation/introduction d'arbres fruitiers dont les fruits seront commercialisés. L'exploitation du bois n'étant pas très courante dans la zone, les arbres forestiers ont tendance à être considérés comme encombrants et donc éliminés. De ce fait, en ce qui concerne les proportions d'arbres conservés, seuls $11 \%$ des arbres associés ont un statut d'arbres conservés, résultat qui contraste avec le pourcentage de $81 \%$ trouvé par Tayo Gamo (2014) à Ngomedzap, et de $91 \%$ trouvé par Temgoua et al. (2018) à Belabo. Quatre espèces inventoriées dans les SAF cacaoyers de l'arrondissenment de Loum sont vulnérables selon les critères de la liste route de l'UICN (2018). Leurs abondances relatives sont cependant faibles, comprises entre 0,17 et $2,77 \%$. Les agriculteurs doivent être davantage encouragés à protégér et à planter ces espèces dans leurs cacaoyères. 


\section{Conclusion}

Cette étude conduite dans les système agroforestiers cacaoyers de Loum dans le littoral Cameroun a permis d'identifier 27 espèces ligneuses appartenant à 23 genres et 18 familles. Des différences significatives ont été enregistrées entre les systèmes agroforestiers cacaoyers des trois zones étudiées, tant au niveau de la richesse spécifique que de la structure et des usages des arbres associés. Les systèmes agroforestiers de la zone de Kwata, où est localisée la réserve forestière de Loum ont présenté une plus grande diversité. En ce qui concerne les usages, les agriculteurs ont principalement orienté leurs cacaoyères vers une diversification de la production. De ce fait, les arbres associés sont majoritairement introduits/conservés à des fins de production fruitière, de la recherche de l'ombrage pour les cacaoyers et des tuteurs pour le poivrier. Cette étude montre que les systèmes agroforestiers cacaoyers de l'arrondissement de Loum ont un bon potentiel de conservation de la biodiversité ligneuse. La présente étude devra être complétée par une évaluation des stocks de carbone.

\section{References}

1. Atangana, D., Khasa, K., Chang, S., \& Degrande, A. (2014). Agroforesterie tropicale. Département de Sciences du bois et de la forêt, Université Laval. 412p.

2. CARPE. (2005). The forest of the Congo Basin: A preliminary assessment. Central African Regional Program for the Environment (CARPE). 37pp.

3. Cissé, A., Aka, J. C. K., Kouamé, D., Vroh, B. T. A., Adou Yao, C. Y., \& N'guessan K. E. 2016.Caracterisation des pratiques agroforestières à base de cacaoyers en zone de forêt dense semidécidue : Cas de la localité de Lakota (Centre-Ouest, Cote d'Ivoire). European Scientific Journal 12 (21): 50-69.

4. Delvaux, B. (1988). Constituants et propriétés de surface des sols dérivés de pyroclastes basaltiques du Cameroun occidental. Approche génétique de leur fertilité. PhD Thesis, UCL, Unité de Chimie des Interfaces, $335 \mathrm{p}$.

5. FAO. (2008). Financial mechanisms for adaptation to and mitigation of climate change in the food and agriculture sectors. Paper presented at the high level conference on world food security: The challenges of climate change and bioenergy, Rome, 3-5 June. 28p.

6. FAO. (2016). State of the World Forests. Rome: FAO.

7. Favrichon, V., Gourlet-Fleury, S., Bar-Hen, A., \& Dessard, H. (1998). Parcelles permanentes de recherche en forêt dense tropicale humide, Eléments pour une méthodologie d'analyse des données. Série Forafri 1998. Document n⿳14. CIRAD. 73 p. 
8. Gockowski, J., \& Sonwa, D. (2010). Cocoa intensification scenarios and their predicted impact on $\mathrm{CO}_{2}$ emissions, biodiversity conservation and rural livelihoods in the Guinea Rain Forest of West Africa. Environmental Management 48: 307-321.

9. Gockowski, J., Tchatat, M., Dondjang, J. P., Hietet, G., \& Fouda, T. (2010). An empirical analysis of the biodiversity and economic returns to cocoa agroforests in southern Cameroon. Journal of Sustainable Forestry 29: 638-670.

10. Hairiah, K., Dewi, S., Agus, F., Velarde, S., Ekadinata, A., Rahayu, S., \& van Noordwijk, M. (2010). Measuring carbon stocks across land use systems: A manual. Bogor, Indonesia. World Agroforestry Centre (ICRAF), SEA Regional Office.

11. Jagoret, P., \& Nyassé, S. (2003). Intensification raisonnée du verger de cacaoyer Camerounais par l'identification des systèmes de productions compétitifs et durables. Rapport annuel scientifique et technique IRAD/ CIRAD, projet Mise au point de systèmes de cacaoculture compétitif et durable en Afrique FSP. 2000. 137p.

12. Jagoret, P. (2011). Analyse et évaluation de systèmes agroforestiers complexes sur le long terme: Application aux systèmes de culture à base de cacaoyer au Centre Cameroun. Thèse de doctorat. Montpellier SupAgro, Montpellier. 288p.

13. Jagoret, P., Michel-Dounias, I., \& Malézieux, E. (2011). Long-term dynamics of cocoa agroforests: a case study in central Cameroon. Agroforestry Systems 81: 267-278.

14. Jagoret, P., Kwesseu, J., Messie, C., Michel-Dounias, I., \& Malézieux, E. (2014). Farmers' assessment of the use value of agrobiodiversity in complex cocoa agroforestry systems in central Cameroon, Agroforestry Systems 88: 983-1000.

15. Kent, M., \& Coker, P. (1992). Vegetation description and analysis: a practical approach. England: John Wiley and Sons Ltd.

16. Kpangui, K. B., Vroh, B. T. A, Gone, Bi Z. B., \& Adou Yao, C. Y. (2015). Diversité floristique et structurale des cacaoyères du V Baoulé: cas de la préfecture de Kokumbo Centre, Côte d'Ivoire. European Scientific Journal 11 (36): 40-60.

17. Lebrun, J. P., \& Stork, A. L. (1991, 1992, 1995, 1997). Enumération des plantes à fleur d'Afrique tropicale, 4 Vols. Genève, Conservatoire et Jardin botaniques de la ville de Genève.

18. Madountsap, T. N., Zapfack, L., Chimi, D. C., Kabelong, B., Tsopmejio, T. I., Forbi, P. F., \& Nasang, J. M. (2017). Biodiversity and carbon stock in the SODECAO Agroforestry System of Center Region of Cameroon: Case of Talba Locality. American Journal of Agriculture and Forestry 5 (4): 121-129. 
19. Manfo, D. A., Tchindjang, M., \& Youta, H. J. (2015). Systèmes agroforestiers et conservation de la biodiversité dans un milieu fortement anthropisé: le cas d'Obala. Revue Scientifique et Technique Forêt et Environnement du Bassin du Congo 5 : 22-34.

20. Martin, D., \& Sieffermann, G. (1966). Le département du Mungo (Ouest-Cameroun). Etude des sols et de leur utilisation. Cahiers ORSTOM. Série Pédologie 4(2):27-49.

21. Mbolo, A. M., Zekeng, J. C., Mala, A. W., Fobane, J. L., Chimi, D. C., Nyako, M. C., \& Tamandjong, V. (2016). The role of Cocoa agroforestry in conserving forest tree diversity in Central Region of Cameroon. Agroforestry. Systems. 90 (4): 577-590.

22. Nair, P. K. R. (1993). An introduction to agroforestry. Klumer Academic Publishers, Dordrecht, Netherlands, 499p.

23. Nair, P. K. R., \& Garrity, D. (2012). Agroforestry, the future of Global land use. Advance in Agroforestry 9, Springer. Dordrecht. 541p

24. Noiha, N. V., Zapfack, L., \& Mbade, L. F. (2015). Biodiversity management and plant dynamic in a cocoa agroforest (Cameroon). International Journal of Plant \& Soil Science 6(2):101-108.

25. Norgrove, L., \& Hauser, S. (2013). Carbon stocks in shaded Theobroma cacao farms and adjacent secondary forests of similar age in Cameroon. Tropical Ecology 54 (1): 15-22.

26. Ramade, F. (1994). Éléments d'écologie. Écologie fondamentale 2. Ediscience international. 594p.

27. Saj, S., Jagoret, P., \& Todem Ngogue, H. (2013). Carbon storage and density dynamics of associated trees in three contrasting Theobroma cacao agroforests of Central Cameroon. Agroforestry Systems 87: 1309-1320.

28. Saj, S., Durot, C., Mvondo-Sakouma, K., Tayo Gamo, K., \& AvanaTientcheu, M. L. (2017). Contribution of companion trees to long-term tree conservation, carbon storage and agroforest sustainability: a functional analysis of the diversity in cacao plantations of Central Cameroon. International Journal of Agricultural Sustainability 15: 282-302.

29. Sonwa, D. J., Nkongmeneck, B. A., Weise, S. F., Tchatat, M., Adesina, A. A., \& Jansens, M. J. J. (2007). Diversity of plants in cocoa agroforests in the humid forest zone of Southern Cameroon. Biodiversity and Conservation 16: 2385-2400.

30. Sunderlin, W. D, Ndoye, O., \& Bikié, H. (2000). Economic crisis, farming systems and forest cover change in the humid forest zone of Cameroon. International Forestry Review 2: 173-181.

31. Tayo Gamo, K. (2014). Dynamique de la biodiversité ligneuse et des stocks de carbone dans les systèmes agroforestiers à base de cacaoyers 
au Centre Cameroun : cas de Ngomezap. Mémoire de fin d'études d'ingénieurs. FASA-Université de Dschang. 106 p.

32. Torquebiau, E. Mary, F., \& Sibelet, N. (2002). Les associations agroforestières et leurs multiples enjeux. Bois et Forêts des Tropiques 271: 23-34.

33. UICN. (2018). The IUCN Red List of threatened species. Version 2018-2. http://www.iucnredlist.org. Downloaded on 10 January 2019.

34. Woodfine, A. (2008). L'adaptation au changement climatiques et l'atténuation de ses effets en Afrique subsaharienne au moyen des pratiques de gestion durable des terres. Terrafrica. 90p.

35. Zapfack, L., Chimi, D. C., Noiha, N. V., Zekeng, J. C., Meyan-ya, D. G. R., \& Tabue, M. R. B. (2016). Correlation between associated trees, cocoa trees and carbon stocks potential in cocoa agroforests of Southern Cameroon. Sustainability in Environment 1(2): 71-84. 